\title{
A multiple transmitter and receiver electromagnetic system for improved target detection
}

\author{
Michal Kolaj ${ }^{1}$ and Richard S. Smith ${ }^{1}$
}

\begin{abstract}
We have developed an alternative strategy for the inevitable deeper inductive electromagnetic (EM) exploration, which will be required as shallow deposits are exhausted. Rather than using very large magnetic moment ground loops, measurement stations are repeated using many smaller sized loops with smaller moments. The multiple transmitter data are then weighted and summed into a single high signal-to-noise ratio $(\mathrm{S} / \mathrm{N})$ composite transmitter. The composite transmitter can be thought of as a postprocessing method that uses the collected multitransmitter data to construct/simulate a transmitter, which maximizes the coupling to a particular target. The appropriate transmitter weights to use will depend on the target location and geometry, and, as such, different weighting schemes allow for the construction of different composite transmitters, each of which will maximally highlight different targets. We have assumed no prior
\end{abstract}

knowledge of the location and orientation of the exploration targets, and we constructed composite transmitters for each possible location of a discretized subsurface and 324 possible target orientations (dipole embedded within a fully resistive medium). A modified difference of squares and a dipole look-up table was used to assess the fit between each composite transmitter and the suggested target location and orientation. Synthetic studies using conductive plate target(s) embedded within a fully resistive medium found that the target locations and orientations could be accurately determined and that the $\mathrm{S} / \mathrm{N}$ of the composite transmitter was significantly higher than that of standard fixed-loop ground and airborne surveys. In a ground time-domain EM field test, 23 transmitter positions were used, and a shallow target (conductive dike) could be identified using the developed methodology. The composite transmitter data we produced was considerably easier to interpret and had a larger amplitude than that of any one single transmitter.

\section{INTRODUCTION}

With the continual depletion of mineral resources, exploration for deeper ore bodies will be essential in sustaining current mine production levels and future demand for resources. The electromagnetic (EM) geophysical prospecting technique is widely used in the exploration for mineral resources because the exploration targets (i.e., the deposit itself, and/or the host structures) are often moderately to highly conductive relative to the background medium. However, from an EM geophysical exploration point of view, deeper ore bodies present technical challenges because the measured responses from these deep ore bodies are small and often below the background noise levels (poor signal-to-noise ratio $[\mathrm{S} / \mathrm{N}]$ ). The main strategy to overcome this issue has been to use large highpowered transmitters with large magnetic moments (Zhdanov,
2010). These high-moment transmitters increase the $\mathrm{S} / \mathrm{N}$ of deeper ore bodies by producing larger magnetic fields at greater depths (Nabighian and Macnae, 1991). However, the logistical issues associated with using transmitter wire loop several kilometers in length and transmitter current generators that are very large and cumbersome are often costly and impractical (Zhdanov, 2010). Furthermore because the drop-off rate of the magnetic field amplitude of a large transmitter loop approaches an inverse cube relation far from the loop, the transmitter moment has to be increased tremendously to have a noticeable impact on the depth of exploration. As an example, increasing the transmitter moment by a factor of two by either doubling the transmitter loop area or the transmitted current would only increase the depth of exploration by roughly $25 \%$.

If the transmitter loop is not positioned properly, such that the magnetic field generated by the transmitter (termed the primary

\footnotetext{
Manuscript received by the Editor 1 October 2014; revised manuscript received 20 January 2015; published online 22 June 2015.

${ }^{1}$ Laurentian University, Department of Earth Sciences, Sudbury, Ontario, Canada. E-mail: mx_kolaj@laurentian.ca; rssmith@laurentian.ca.

(C) 2015 Society of Exploration Geophysicists. All rights reserved.
} 
magnetic field $\mathbf{H}^{P}$ ) does not couple well with the target (i.e., it does not crosscut the target), then the amount of induced current within that body will be greatly reduced (Faraday's law) and the resultant magnetic field (termed the secondary magnetic field $\mathbf{H}^{S}$ ) generated by those induced currents (via Ampere's law) may be small enough to be undetectable (i.e., low $\mathrm{S} / \mathrm{N}$ ). It is thus common practice to move the transmitter loop to one or more other locations to ensure adequate coupling, which will add to the logistical challenges (Nabighian and Macnae, 1991). In airborne EM, the coupling issue is less prevalent due to the moving transmitter loop, but the size of the transmitter, and thus the depth of penetration of the system, is limited because the transmitter moment is restricted by the size and power of the aircraft (Palacky and West, 1991). In the ground and airborne situations, longer recording times are preferable because the waveform stacking process increases the S/N. However, a longer stacking time results in decreased production in ground surveys (Zhdanov, 2010) and is limited by the speed of the aircraft in airborne surveys.

An alternative strategy, presented by Lymburner and Smith (2015), which aims to address the issues of depth of penetration and coupling for the exploration of deeper ore bodies, is to repeat the profile or grid with many, potentially smaller, transmitters. In typical EM data processing, when multiple transmitter locations are used, the responses are generally not combined. Each transmitter will couple differently to the target(s), may possess different informations, and will have a different S/Ns. As such, the multiple transmitter data are generally interpreted semi-independently with the goal in mind of producing one model that fits all available data. However, when a large number of transmitters are used (10s-100s), interpreting each transmitter is not efficient and/or feasible in a reasonable amount of time. Because the EM problem is linear, we can combine the measurements from each transmitter into a single measurement, which represents the EM response to a very large moment "composite transmitter." The large moment of the composite transmitter will ensure a depth of exploration superior to that of traditional EM surveying. The ideal manner in which the multiple transmitter data are combined into one large composite transmitter will depend on the location and orientation of the target(s) because each single transmitter will couple to the target(s) differently. Thus, the multiple transmitter data can be summed into different composite transmitters, each of which highlights different targets by maximizing the coupling between the composite transmitter and the target. Similar processing techniques and multifold transmitter-receiver arrays for deep multiconductor exploration have been discussed in Polzer et al. (1989), Powell (1990), and Powell et al. (2007). The critical difference between the previous methods and the one discussed in this paper is the move from roughly three transmitter positions to 10 or even $100 \mathrm{~s}$ of positions. Multiple transmitter and receiver systems have also been used in the detection and characterization of unexploded ordinances whereby an array of multiaxis transmitters and receivers was found to provide superior location and property information (Snyder and Oden, 2012). These systems typically include one or several single axis (typically $z$ directed) or three-orthogonal component transmitters and an array of receivers (single $z$-axis or three-orthogonal component) mounted on a small rigid movable platform.

We begin by discussing the multiple transmitter data summation process and how it can increase the $\mathrm{S} / \mathrm{N}$ from deeper targets compared with traditional methods. With synthetic studies, we present a simple imaging procedure that can identify the depth and orientation of the target(s) and the appropriate composite transmitter(s) that maximizes the $\mathrm{S} / \mathrm{N}$ for the identified target(s). This method is also successfully tested on the multiple transmitter EM data of Lymburner and Smith (2015), which were collected over a shallow conductor on Wallbridge mining property in the east range of the Sudbury Basin in Ontario, Canada.

\section{METHODOLOGY}

The goal of the method presented here is to sum the different transmitter responses in such a way as to maximally enhance the response from a certain target to achieve a larger S/N. One way of summing the transmitters is to apply weights that are proportional to how well the transmitters coupled to the target (Lymburner and Smith, 2015). To efficiently calculate the coupling between the transmitter and the target, we use a dipole approximation for the transmitter and the target:

$$
\begin{aligned}
C\left(\mathbf{r}_{t}, \mathbf{r}_{d}, \mathbf{m}_{\theta}\right)= & \frac{M_{t}}{4 \pi\left|\mathbf{r}_{t}-\mathbf{r}_{d}\right|^{3}} \\
& \times\left[\frac{3 \mathbf{m}_{\theta} \cdot\left(\mathbf{r}_{t}-\mathbf{r}_{d}\right)}{\left|\left(\mathbf{r}_{t}-\mathbf{r}_{d}\right)\right|^{2}}\left(r_{t}-r_{d}\right)-m_{\theta}\right],
\end{aligned}
$$

where $C$ is the coupling coefficient between a transmitter located at $\mathbf{r}_{t}$ of dipole moment $M_{t}$ (effective area multiplied by the transmitted current) and a dipole target of orientation $\mathbf{m}_{\theta}$ (unit vector normal to the planar target) located at $\mathbf{r}_{d}$. The symbol $\|$ represents the magnitude of the enclosed vector. Because the dipole formula (equation 1) is typically expressed as a vector equation, we set the variables $r_{t}, r_{d}$, and $m_{\theta}$ to represent the component of $\mathbf{r}_{t}, \mathbf{r}_{d}$, and $\mathbf{m}_{\theta}$, respectively, parallel to the axis of the transmitter, such that equation 1 results in a scalar value (i.e., the coupling coefficient between the dipole transmitter and target). When multiple transmitters are present, $C$ is generally divided by the maximum value (maximum coupled transmitter) to be a dimensionless scalar ranging from \pm 1 (termed the normalized coupling coefficient). Because the method approximates the target with that of an equivalent dipole, it is only valid for discrete targets. Nondipole transmitters could also be used whereby equation 1 would need to be replaced with the appropriate formula for the particular transmitter geometry (i.e., for an arbitrary transmitter wire loop, the coupling coefficient could be calculated by the Biot-Savart law to calculate the component of the magnetic field normal to the target at the targets location).

Figure 1 displays the normalized coupling coefficient $C$ for vertical dipole transmitters (spaced $50 \mathrm{~m}$ apart) and a target with varying dip located in the center of the profile at a depth of $500 \mathrm{~m}$. The normalized coefficients seen on the $y$-axis are the weighting factors applied to the corresponding data associated with the transmitter at that location. This ensures that the data associated with transmitters that coupled well to the target (high $\mathrm{S} / \mathrm{N}$ ) are enhanced and those that did not couple well (low $\mathrm{S} / \mathrm{N}$ ) are reduced. However, the weights vary for different target locations and orientations, and as such, the target orientation and location must be known, estimated, or assumed to apply the appropriate weights, so as to enhance the response. For example, for a vertical target (Figure 1, solid line), the transmitters located at -250 and $250 \mathrm{~m}$ would be weighted the highest (weights of -1 and +1 , respectively). 
However, if the dip of the target was $30^{\circ}$, then those same transmitter positions should have weights of 0.24 and 0.71 (Figure 1, dashed-dotted line). Throughout this work, the convention for orientation (strike and dip) is on a $\left(0^{\circ}\right.$ and $\left.180^{\circ}\right)$ interval, whereby a $0^{\circ}$ or $90^{\circ}$ strike would imply a north-south- or east-west-trending feature, respectively. The dip angle is measured from the east-facing side of the target (with the exception of a $90^{\circ}$ strike, whereby it is measured from the south).

The proposed method is to assume every possible target location $\left(\mathbf{r}_{d}\right)$ and orientation $\left(\mathbf{m}_{\theta}\right)$ and to sum the multiple transmitter data for each assumed location/orientation using the appropriate dipole coupling coefficients ( $C$, equation 1$)$. When the assumed location and orientation match the actual location and orientation of the target, the summed data will maximally reinforce the signal from that particular target. The weighted sum of the transmitters will form an equivalent composite transmitter, which will be the optimal transmitter (enhanced $\mathrm{S} / \mathrm{N}$ ) for that particular target. This can be assessed quantitatively by a comparison of the shape of the profile with the shape from a theoretical body at that location (dipole look-up table). In this paper, the fit is determined using a modification of the difference-of-squares method from Smith and Salem (2007):

$$
I_{\left(\mathbf{r}_{d}, \mathbf{m}_{\theta}\right)}=\left\{1-\frac{\sum\left[\left(\mathbf{H}^{S} \mathbf{C}_{\left(\mathbf{r}_{d}, \mathbf{m}_{\theta}\right)}-\mathbf{L}_{\left(\mathbf{r}_{d}, \mathbf{m}_{\theta}\right)}\right]^{2}\right.}{\sum\left[\mathbf{L}_{\left(\mathbf{r}_{d}, \mathbf{m}_{\theta}\right)}\right]^{2}}\right\}^{2},
$$

where $I$ is the degree of fit, $\mathbf{H}^{S}$ is a matrix of the survey data (time derivative or magnetic field measurements), where each row represents a different station location (at a particular frequency or time channel) and each column represents a different transmitter location, $\mathbf{C}$ is the vector of coupling coefficients calculated for each transmitter position, and $\mathbf{L}$ is the corresponding look-up data for a dipole of orientation $\mathbf{m}_{\theta}$ located at $\mathbf{r}_{d}$ for the same station locations as $\mathbf{H}^{S}$. The dipole look-up data $(\mathbf{L})$ are calculated using the dipole equation (Appendix A) for a fixed number of subsurface locations and dipole orientations. In this work, the orientation (strike and dip of the dipole) varied from $0^{\circ}$ to $170^{\circ}$ in $10^{\circ}$ intervals (324 possible dipole orientations), and the discretized subsurface cell size (i.e., location of the dipoles) varied from example to example. As the discretization (for the orientation and subsurface) is made finer, the data fit is generally improved, but there is an increase in computation time. Note that each column of $\mathbf{H}^{S}$, and $\mathbf{C}$, and $\mathbf{L}$ are normalized to unit amplitudes. The value of $I$ is set to zero when negative, and when multiple EM component data are available, $I$ is the product of the fit for each of the measured components (i.e., for three-component EM data, $I=I_{x} I_{y} I_{z}$ ). When the fit $I$

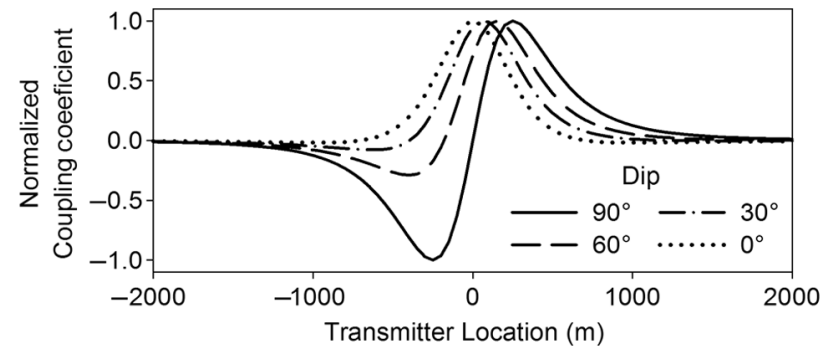

Figure 1. Normalized coupling coefficient as calculated using equation 1 between a vertical dipole transmitter and a dipole target of varying dip located in the center of the profile at a depth of $500 \mathrm{~m}$. is close to unity, there is a high likelihood that there is a target at that $\mathbf{r}_{d}$ with orientation $\mathbf{m}_{\theta}$. Equation 2 is solved by finding the maximum $I$ values over a discretized subsurface $\left(\mathbf{r}_{d}\right)$ with dipoles of varying orientations $\left(\mathbf{m}_{\theta}\right)$. From this, an image section (or volume) of the most likely dipole locations and orientations ( $\mathbf{r}_{d}$ and $\mathbf{m}_{\theta}$ values) that fit the data can be produced. Alternatively, equation 2 could be inverted to find the location and orientation that give maximum $I$ values.

In practice, $I$ is calculated over a variable window size, so as to not degrade the fit value due to areas distant from the target, which will likely have low S/N. A variable window size is also necessary when multiple targets are present, so as to fit multiple dipoles in lieu of a single dipole for the entire survey area. The window size is the distance from the peak amplitude of the look-up table data to the minimum distance, which encompasses a user-defined percentage (relative term $\alpha$ ) of the total sum of the look-up table data (the magnitude of the look-up data is used when multiple components are being used to calculate equation 2). The window size is calculated for each look-up table target/dipole. Because the footprint (the lateral extent of the fields emanating from the target) increases as the target becomes deeper, the window size would similarly increase.

Once the target locations and orientations are known, the appropriate coupling coefficient vector $\mathbf{C}$ can be selected. The multitransmitter data can then be summed (via $\mathbf{H}^{S} \mathbf{C}$ ) to form the optimal composite transmitter, which provides the maximum $\mathrm{S} / \mathrm{N}$ for the identified target(s). Note that when multiple targets are present, there will be an optimal composite transmitter for each target (different C).

\section{SYNTHETIC EXAMPLES}

Because the imaging and transmitter summation algorithm (equation 2) is general and includes no time/frequency variable, frequency-domain surveys were simulated for computational simplicity, and only the quadrature component was considered. The synthetic examples were generated in GeoTutor (PetRos EiKon) using the VHPlate algorithm (Walker and West, 1991) for conductive plates embedded in a completely resistive host.

\section{Example 1 - Hybrid ground/air survey}

The described survey methodology and logistics (i.e., repeating profiles with multiple transmitter positions) are best exploited with a hybrid airborne/ground EM system. With a typical airborne EM transmitter and a distributed ground receiver array system, the survey time is drastically reduced as compared with performing the survey using many ground loop positions and a single roving receiver. A single-frequency $(100 \mathrm{~Hz}), 2$ million $\mathrm{Am}^{2}$ dipole moment airborne transmitter at a height of $120 \mathrm{~m}$ with ground receiver stations (three-component) spaced every $100 \mathrm{~m}$ along a $3-\times 3-\mathrm{km}$ grid is simulated. Dipole moments of 2 million $\mathrm{Am}^{2}$ are typical for airborne systems, e.g., MEGATEM 2,100,000 Am² (Smith et al., 2003), HELITEM 2,000,000 $\mathrm{Am}^{2}$, and VTEM 2,500,000 $\mathrm{Am}^{2}$ (Smith and Volkovitsky, 2014). Due to computational limits, the transmitter locations are spaced every $200 \mathrm{~m}$, resulting in a net total of 256 transmitters each with 961 three-component magnetic field recordings. The top center of a $100-\mathrm{S}$ plate is located at $(0,0$, $-500 \mathrm{~m}$ ). The plate has a strike and dip of $40^{\circ}$ and $30^{\circ}$, respectively, and has a strike length and downdip length of 300 and $150 \mathrm{~m}$, respectively. In addition to the hybrid airborne/ground survey, two 
other single-frequency surveys $(100 \mathrm{~Hz})$ were simulated for comparison purposes: a large horizontal $3-\times 1.5-\mathrm{km}$ fixed-loop ground survey (100 million $\mathrm{Am}^{2}$ equivalent dipole moment transmitter and a roving three-component receiver) and an airborne survey (threecomponent receiver is towed $130 \mathrm{~m}$ behind and $50 \mathrm{~m}$ below the 2 million $\mathrm{Am}^{2}$ airborne transmitter). An aerial view of the survey geometry can be seen in Figure 2. Gaussian noise was added to the synthetic survey data but because multiple acquisition systems were simulated (ground, airborne, and semiairborne), different noise values were used. Following the results of Smith et al. (2001), we assigned the highest noise level ( $0.1 \mathrm{pT})$ for the airborne system. A noise level of $40 \%$ of the airborne system was assigned for the fixed-loop ground survey $(0.04 \mathrm{pT})$, and because the hybrid air-ground system is a mixture of both survey types (ground and air), we assigned it a noise value in between the other two systems $(0.07 \mathrm{pT})$. The window size for the dipole look-up table is the same size as the survey area (i.e., $\alpha=100 \%$; the dipole look-up table comparison in equation 2 is performed over the entire survey area).

For computational efficiency, the imaging algorithm (equation 2) was first run at a coarse discretization over the entire survey area and for a large depth range. Once the general location of the suspected target was found, it was rerun at a finer discretization to produce Figure $3(25-\times 25-\times 50-\mathrm{m}$ cell size $)$. In Figure 3 , the locations correspond to $\mathbf{r}_{d}$, the color saturation corresponds to the fit value $I$ scaled to be between 0 and 1 , and the hue corresponds to the most likely dip (left panels) or strike (right panels) of the target (derived from $\mathbf{m}_{\theta}$ ). When $I$ was less than 0.5 , the color was set to white. The strike and dip were both discretized from $0^{\circ}$ to $170^{\circ}$ in $10^{\circ}$ intervals. A strike of $0^{\circ}$ corresponds to a north-south target, whereas a strike of $90^{\circ}$ would correspond to an east-west-striking target. A dip of less than $90^{\circ}$ implies that the structure is dipping toward the east, whereas a dip greater than $90^{\circ}$ implies a dip toward the west.

As can be seen in Figure 3, the estimated location of the plate matches the actual location. Most of the $I$ values greater than 0.5 ranged from $20^{\circ}$ to $60^{\circ}$ for the strike, and, from $30^{\circ}$ to $60^{\circ}$ for the dip. The fit that produced the maximum $I$ value corre-

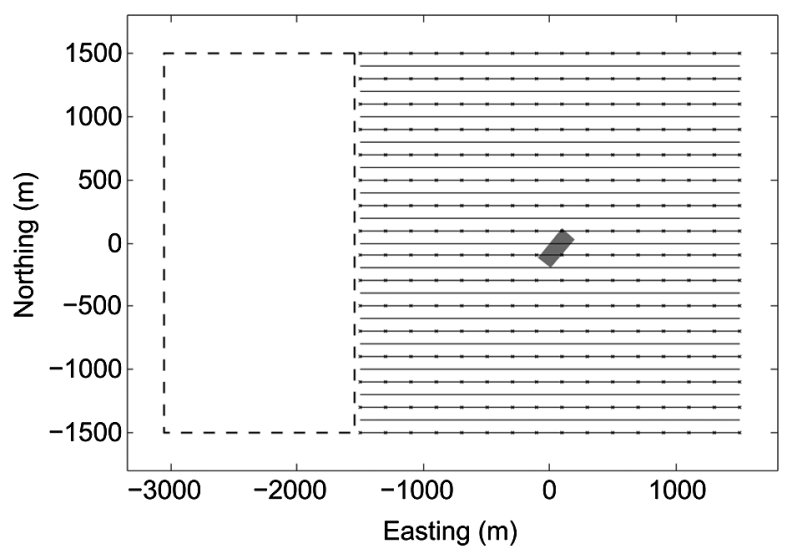

Figure 2. Plan view of the survey geometry used in the first synthetic example. Black solid lines indicate the locations of the threecomponent receivers (spaced $100 \mathrm{~m}$ apart) on a $3-\times 3-\mathrm{km}$ grid. An $\mathrm{x}$ indicates the locations of the airborne transmitter, and the dashed black line indicates the location of the ground transmitter loop. The $300-\times 150-\mathrm{m}$ plate, shown in gray, is buried $500 \mathrm{~m}$ below the surface. sponded to a dipole located at $(50,-50$, and $550 \mathrm{~m})$ with a strike and dip of $40^{\circ}$ and $30^{\circ}$, respectively. For reference, the center point of the actual plate was located at $(50,-42$, and $538 \mathrm{~m})$. The excellent degree of fit can be explained via Figure 4, which depicts the response of the large plate and that of the best-fitting dipole. It is clear that the dipole model is an adequate approximation for this
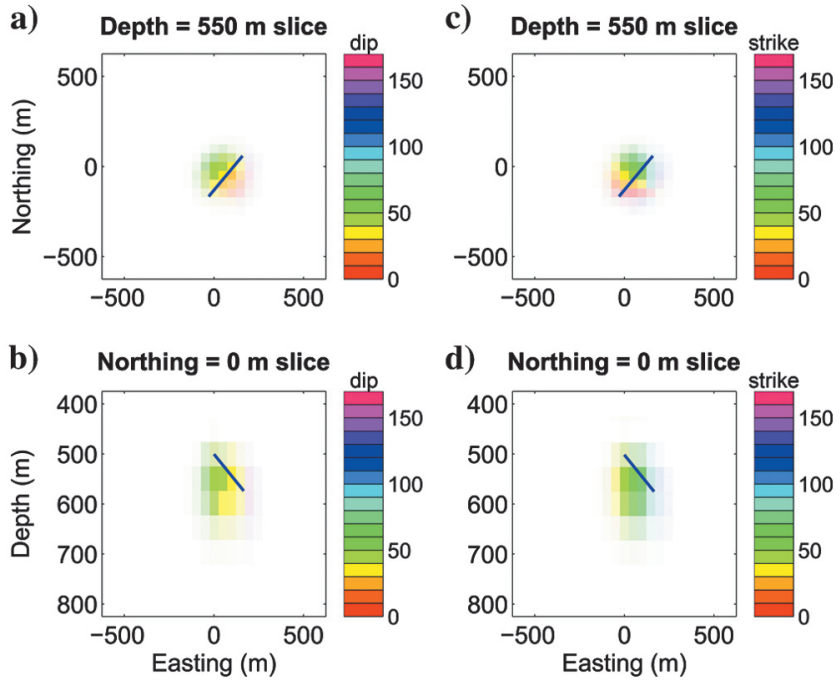

Figure 3. (a and c) Horizontal slice plan view at depth $=550 \mathrm{~m}$, and vertical slice section view at northing $=0 \mathrm{~m}$ with the (b) dip and (d) strike calculated using equation 2. The dark-blue line corresponds to the location of the plate target (intersection of the plate and the slice plane). The cell size is $25-\times 25-\times 50-\mathrm{m}$.
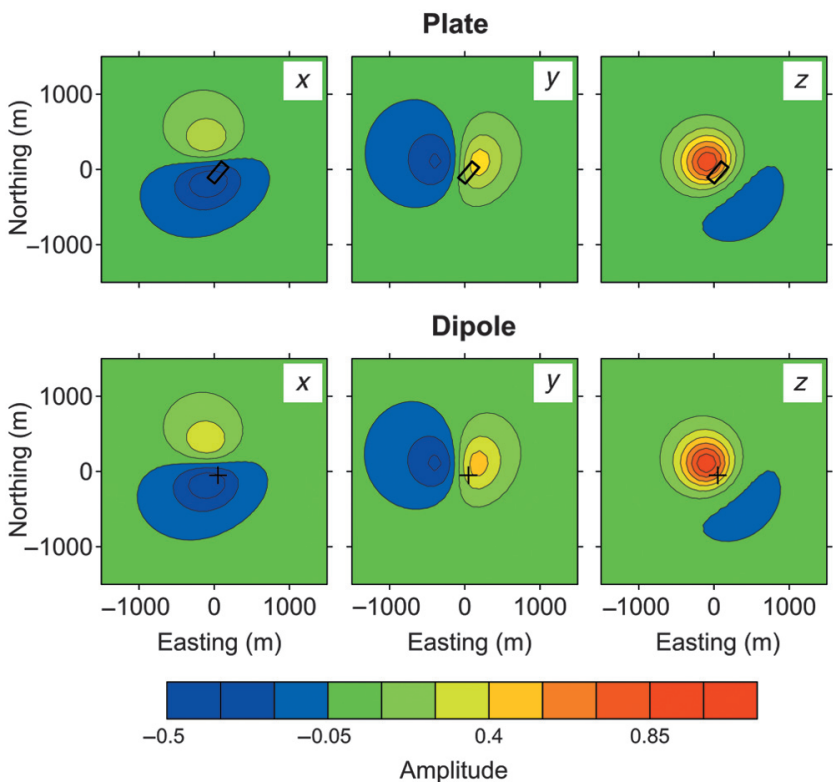

Figure 4. Plan view comparison of the three-component magnetic field $\left(\mathbf{H}^{s}\right)$ synthetic plate data (a well-coupled transmitter; no noise is present, and the plate location is shown with a black rectangle) and best-fitting dipole model for the same transmitter (the dipole location is shown with + ). Data from both models were normalized by the maximum value. 
plate target and that equation 2 can recover a dipole model that explains the measured data.

Because the location and orientation of the target can now be estimated (maximum $I$ value), the multiple transmitter data can be summed $\left(\mathbf{H}^{S} \mathbf{C}\right.$, equation 2$)$ to form a composite transmitter. By summing the single transmitter data using weights calculated based on the coupling between the target and the transmitters, the response from that target will be maximally enhanced. This particular composite transmitter will be considered optimal for the target located at $(50,-50$, and $550 \mathrm{~m})$ with a strike and dip of $40^{\circ}$ and $30^{\circ}$, respectively. A comparison of the data from the optimal composite transmitter, the fixed-loop ground, and the airborne survey data using the survey geometry from Figure 2 can be seen in Figure 5. Note the amplitude of the composite transmitter is roughly 13 times larger than that of the fixed-loop ground and the airborne surveys (Figure 5a). The S/Ns for the composite transmitter, fixedloop ground survey, and airborne survey are 64,24 , and 11 , respectively. In the fixed-loop system, if the loop is moved to a different location, the coupling between the transmitter and the target will change and this may either increase or decrease the amplitude of the response and the resulting $\mathrm{S} / \mathrm{N}$. As a direct result, the survey will be biased toward targets/features that coupled well to the transmitter. With a multitransmitter survey, the potential combinations in which the composite transmitter can be constructed significantly removes this bias because no features/targets will be in a nullcoupled geometry. Furthermore, with typical aircraft speeds, the equivalent transmitter spacing could be as low as roughly $15 \mathrm{~m}$ (as opposed to $200 \mathrm{~m}$, which is used in this example), which would have resulted in 24 times the number of transmitter locations. Even by a higher estimate for noise (airborne survey noise level of $0.1 \mathrm{pT}$ )
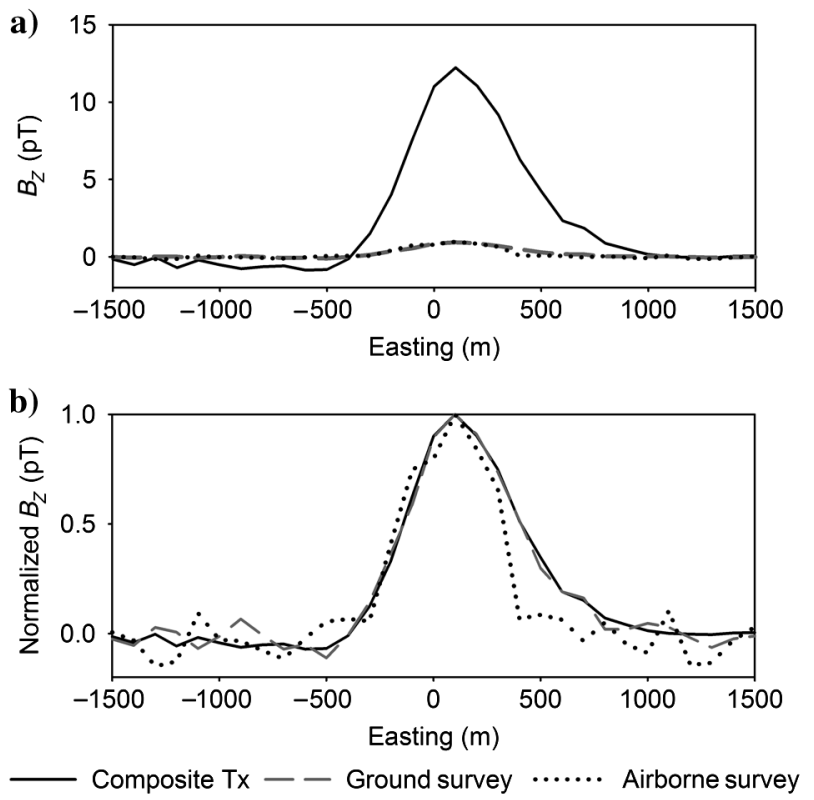

Figure 5. Comparison of the center line (northing $=0 \mathrm{~m}$ ) of the summed composite transmitter (solid black line), fixed-loop ground survey (dashed gray line), and the airborne survey (dotted black line). (a) Comparison of the amplitudes of the responses. (b) Data from each system are normalized to a maximum signal of one, so that the relative noise can be seen in the background. this would have resulted in an S/N of approximately 220 for the composite transmitter of the hybrid survey.

\section{Example 2 - Multiple target ground survey}

In the second example, a single-line survey with 81 ground stations and transmitters $\left(100 \mathrm{~Hz}\right.$ operating frequency and $10^{6} \mathrm{Am}^{2}$ moment dipole transmitters) on a $4-\mathrm{km}$ line was simulated. Two $300 \mathrm{~m}$ (strike length) $\times 150 \mathrm{~m}$ (dip length) plate targets were used. Plate 1 was centered at $(-700,0$, and $-175 \mathrm{~m})$ with a strike and dip of $45^{\circ}$ and $90^{\circ}$, respectively, and plate 2 was centered at $(746,46$, and $-188 \mathrm{~m}$ ) with a strike and dip of $135^{\circ}$ and $30^{\circ}$, respectively. A view of the survey geometry can be seen in Figure 6 .

Figure 7 was generated using equation 2 with a $25-\times 25-\times 25-\mathrm{m}$ cell size. An $\alpha$ of $80 \%$ was used to avoid cross-contamination of the signals from the two targets when performing the dipole look-up table comparison. Recall that an $\alpha$ value

a)

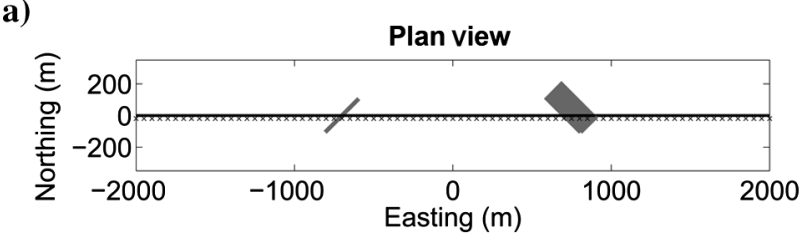

b)

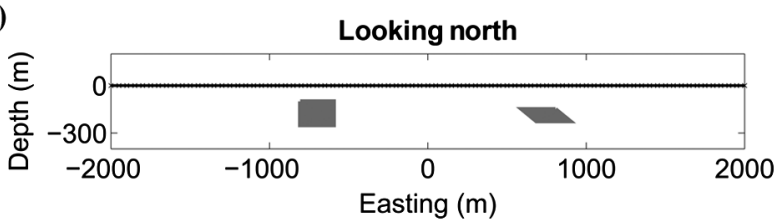

Figure 6. (a) Plan view of the survey geometry and (b) side view used in the second synthetic example. The black solid line indicates the surveyed profile of the three-component receivers (spaced $50 \mathrm{~m}$ apart and 4-km line). An $\mathrm{x}$ indicates the location of the ground dipole transmitter (spaced $50 \mathrm{~m}$ apart and $20 \mathrm{~m}$ south of the receiver line). The two $300-\times 150-\mathrm{m}$ plates projected onto the plan and section are shown in gray.
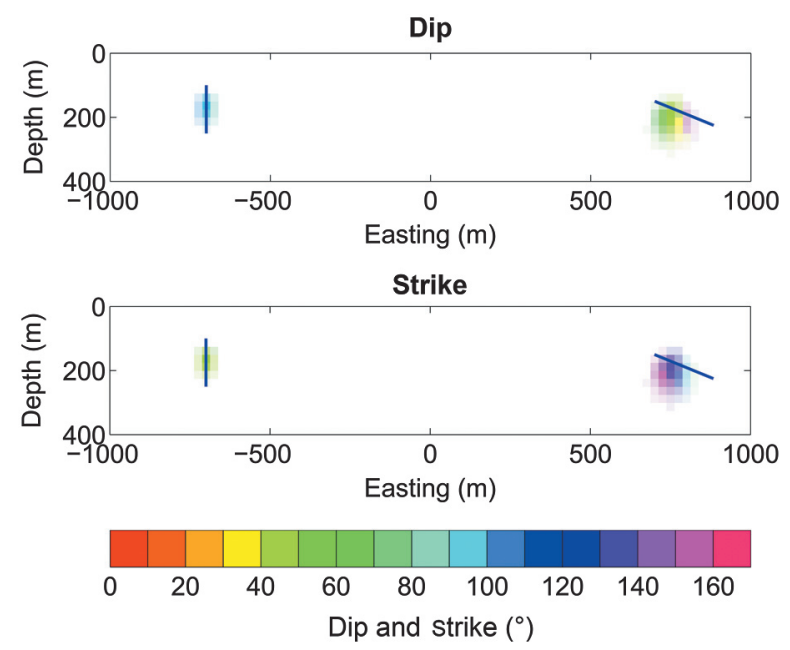

Figure 7. Equation 2 calculated for the two-plate synthetic survey depicted in Figure 6. The northing is equal to $0 \mathrm{~m}$ across the profiles. The dark-blue lines correspond to the intersection of the two plate targets and the section. The cell size is $25 \times 25 \mathrm{~m}$. 
below $100 \%$ implies that the dipole table look-up is not performed using all receiver/station locations but rather using only stations that encompass most of the signal (80\% in this case) of the particular look-up dipole. The two resolved targets are best fit with dipoles located at $(-700,0$, and $175 \mathrm{~m})$ with a strike and dip of $40^{\circ}$ and $90^{\circ}$, respectively, and at $(750,0$, and $-200 \mathrm{~m})$ with a strike and dip of $140^{\circ}$ and $40^{\circ}$, respectively. The best-fitting dipoles match well with the actual locations and orientations of the plates. As in example 1, once the location and orientation of the target are known, the optimal composite transmitter can be constructed via $\mathbf{H}^{S} \mathbf{C}$, where $\mathbf{C}$ contains the transmitter-to-target coupling factors for the identified targets. Because there were two targets identified in this example, there will be two different coupling vectors (i.e., two composite transmitters). Many of the transmitter positions coupled well to both targets, and, as such, the measured magnetic field data detect both deposits (Figure 8a). However, by the optimal composite transmitters, both targets can be separated into their individual responses (Figure $8 \mathrm{~b}$ and $8 \mathrm{c}$ ). Two of the most strongly coupling single transmitter response profiles that couple weakly to the other conductor were manually selected for comparison and are shown in Figure 8 (dashed lines). Note that the composite transmitters provide significantly larger response amplitudes than the strongly coupled single transmitters. Furthermore, the likelihood

a)
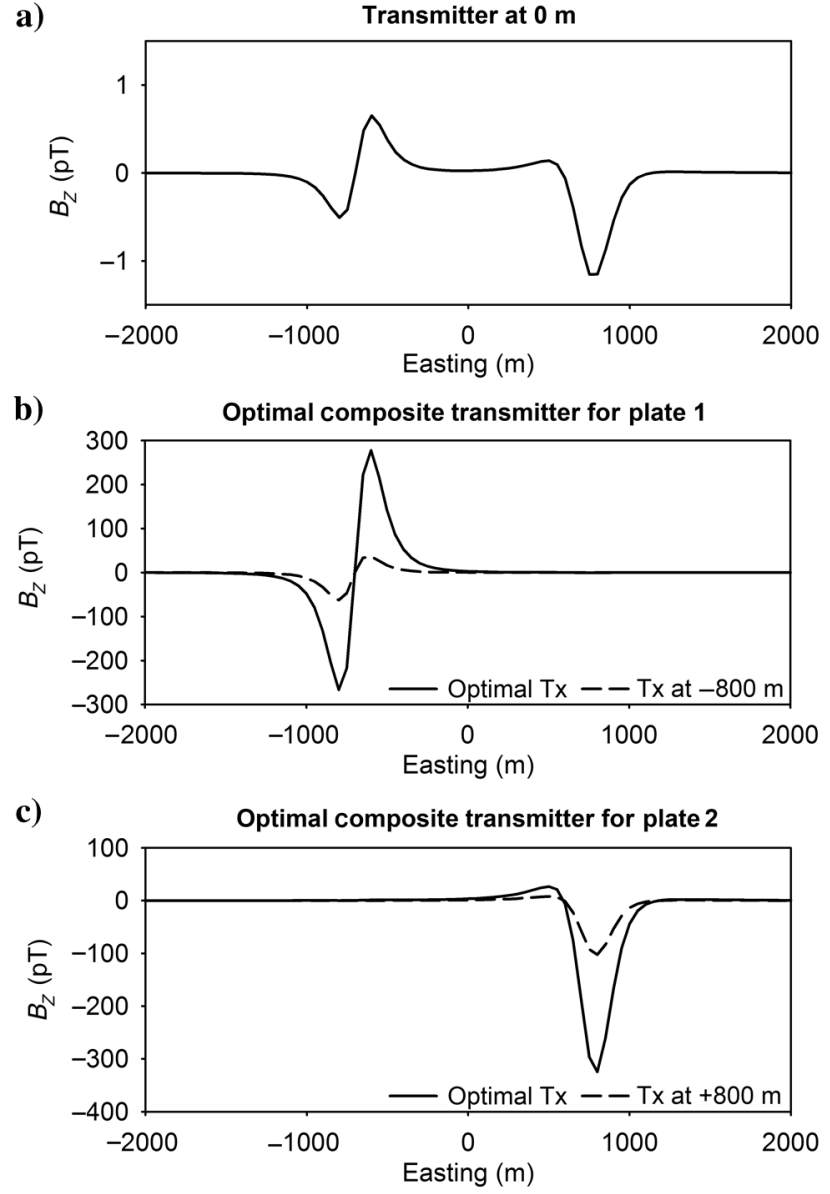

Figure 8. (a) Comparison of single-transmitter (Tx) profiles (dashed line in panels $[b$ and $c]$ ) with that of the optimal composite transmitters (solid line, one for each target identified in Figure 7) for the survey depicted in Figure 6. of manually positioning the single transmitters in the ideal locations is unrealistic unless the targets are already well understood.

\section{FIELD EXAMPLE}

A test time-domain EM survey was conducted over a small, thin dike in the northeast range of the Sudbury Basin and is discussed in more detail in Lymburner and Smith (2015). Based on previous geophysical work and drilling and geologic information, the near-surface target is believed to be at a depth of approximately $60-120 \mathrm{~m}$, trending at an azimuth of $33^{\circ}$ and is vertical to subvertical. Although this is a relatively shallow target, it was easily accessible and could act as a relatively easy target to validate the methodology. The test survey was conducted over a $1-\mathrm{km}$ line with a station spacing of $25 \mathrm{~m}$ (three-component receiver coils). The $30-\mathrm{Hz}$ transmitters (440- $\mu$ s ramp turn-off, 20,000-Am ${ }^{2}$ moment, $10-\times 10-\mathrm{m}$ loop with 10 turns at $20 \mathrm{~A}$ ), spaced every $25 \mathrm{~m}$, occupied the inner $550 \mathrm{~m}$ of the line for a total of 23 transmitter positions. Stations in close proximity to the transmitter ( $\pm 50 \mathrm{~m}$ from the transmitter) contained no discernible signal. This is thought to be due to saturation of the receiver (analog-to-digital converter) causing the readings to be corrupted (Lymburner and Smith, 2015). As such, these stations were removed during the data editing stage. The data from the $z$-component for a poorly coupled transmitter and a well-coupled transmitter can be seen in Figure 9. Note that the well-coupled transmitter $(-150 \mathrm{~m})$ has larger amplitudes, but a significant portion of the profile had to be removed due to corruption.

Because the peak-to-peak distance in the $z$-component of the anomalous response was only roughly $75 \mathrm{~m}$ (Figure 9), most of
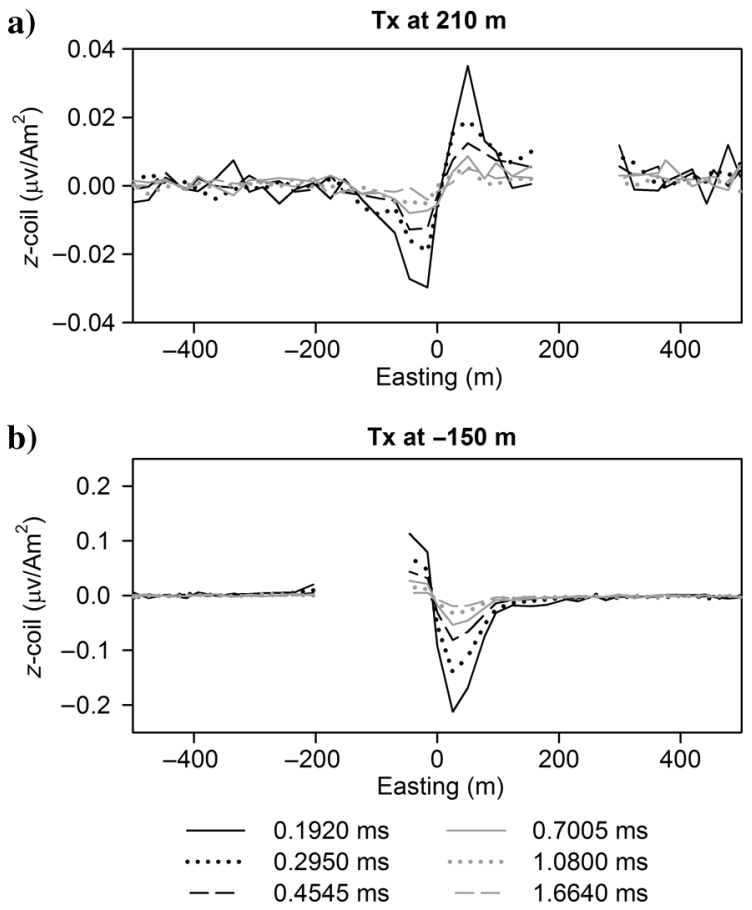

Figure 9. Off-time data collected overtop a shallow conductive dike for two transmitter positions, where the corrupted data $( \pm 50 \mathrm{~m}$ from the transmitter position) have been removed. (a) Poorly coupled, low-S/N transmitter (Tx) that shows the full anomalous response. (b) Well-coupled, high-S/N transmitter in which the western portion of the anomalous response is missing. 
the well-coupled transmitters had a large portion of the anomalous/ target response removed. Apart from the very low $\mathrm{S} / \mathrm{N}$ data from transmitters on either ends of the line (Figure 9), no single transmitter contained the full response of the target. However, equation 2 will still produce a meaningful result because the sum of all of the transmitter positions contains the full response of the target (Lymburner and Smith, 2015). The look-up table in equation 2 (L) was modified to include the effect of having missing portions of the data in proximity to the transmitter positions. It was found that by removing many of the station positions, the solution had a bias toward shallow dipping near-surface targets. This effect was avoided by fitting only steeply dipping dipoles $\left( \pm 30^{\circ}\right.$ from vertical) and decreasing the contribution from the $y$-component, which had the lowest $\mathrm{S} / \mathrm{N}$. Because the existing geologic information and distal transmitters (in which the data corruption does not coincide with the anomalous response; Figure 9a) suggest a steeply dipping body, this restriction was justified. The estimate of the target location and orientation at an intermediate time channel can be seen in Figure 10.

The maximum $I$ value in Figure 10 is at $(10,10$, and $70 \mathrm{~m})$ at a strike and dip of $30^{\circ}$ and $70^{\circ}$, respectively, which matches the suspected location and orientation of the body. Repeating the algorithm on progressively later time channels revealed that the peak $I$ value deepened slightly (from 60 to $90 \mathrm{~m}$ ) and the dip became slightly steeper (from $70^{\circ}$ to $90^{\circ}$ ). This may represent the migration of currents with time into a more conductive portion of the body. Because the location and orientation of the target can now be estimated, the optimal composite transmitter can be constructed to form the high$\mathrm{S} / \mathrm{N}$ data (Figure 11a and 11c, solid black line). For comparison, the look-up table $(\mathbf{L})$ response is also shown (Figure 11a and $11 \mathrm{c}$,
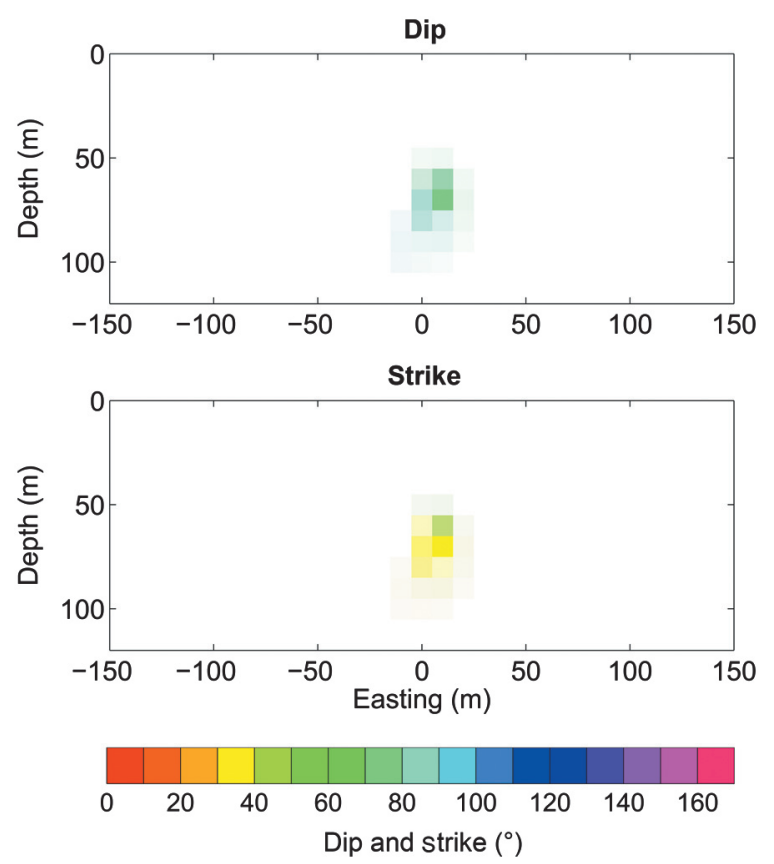

Figure 10. Equation $2(\alpha=100 \%)$ calculated at an intermediate time channel over the field target (suspected to be at a depth of approximately $60-120 \mathrm{~m}$, trending at an azimuth of $33^{\circ}$ and is vertical to subvertical). The northing is equal to $10 \mathrm{~m}$ across the profile (i.e., the best-fitting target is $10 \mathrm{~m}$ north of the surveyed profile). Each cell size is $10 \times 10 \mathrm{~m}$. dashed gray line). Note that although the composite transmitter response appears irregular (especially toward the east in the $z$ - and $x$ components), the irregularity is in fact expected as is also seen in the look-up table profile. This irregularity is due to the data removed due to corruption (missing data adjacent to the transmitter locations), and the good fit between the composite transmitter and the look-up data supports the validity of our method, which incorporates the corrupted data into our dipole look-up table model.

Because the body is relatively shallow, only the transmitters in close proximity to the center of the profile contributed significantly to the composite transmitter. As such, the relative increase in the signal amplitude using the composite transmitter is not as high as it was in the synthetic examples. However, in this example, interpreting the composite transmitter signal (Figure 11a and 11c) is significantly easier than that of any single transmitter location (Figures $11 \mathrm{~b}, 11 \mathrm{~d}$, or $9 \mathrm{~b}$ ). The single transmitter locations only show half of the expected anomalous response due to the removal of the corrupted data. Any attempt to interpret the corrupted single transmitter data would have presented significant ambiguity as to the location and orientation of the target.

\section{DISCUSSION}

Multitransmitter data ensure adequate coupling and allow for the construction of a high-S/N composite transmitter. The construction of the composite transmitter requires specifying the coupling between the transmitters and target, and as such, knowledge of the target location and orientation is essential. In this work, we solve this issue by assuming that the target response can be approximated with a dipole embedded within a fully resistive medium and that the coupling between the transmitter and the target can be approximated using the coupling between two dipoles. The dipole approximation has been used frequently in many areas of EM geophysics (e.g., Pasion and Oldenburg, 2001; Sattel and Reid, 2006; Smith and Salem, 2007). The dipole approximation will be valid for dis- a)
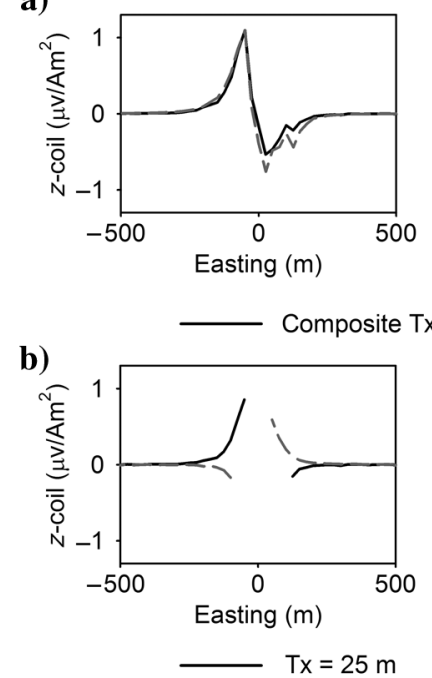

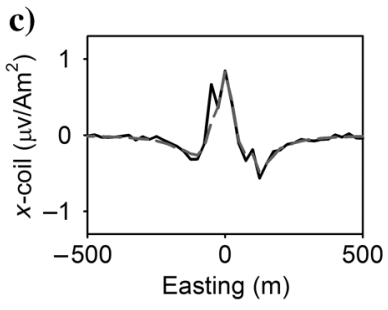

c)

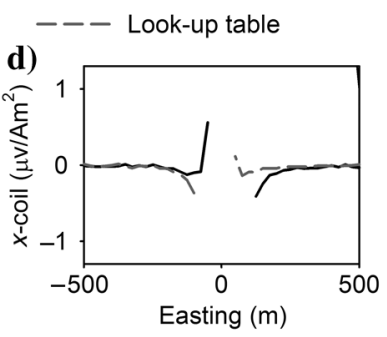

Figure 11. Comparison of the composite transmitter (Tx) response and that of the look-up table for the (a) $z$ - and (c) $x$-components. Two well-coupled single transmitter profiles showing the extent of data corruption for the (b) $z$ - and (d) $x$-components. 
crete targets, so long as the survey dimensions are large relative to the size and depth of the body. The dipole assumption will break down for large and shallow targets, and apart from forward modeling, it is difficult to predict when the approximation will fail. In general, the approximation breaks down gradually and begins by fitting a dipole deeper than the actual position of the target. Moreover, equation 2 attempts to fit a single dipole to the data (or a section if windowing is used). If multiple close bodies are present, there will be an interaction between the two bodies and a superposition of their respective fields. Equation 2 minimizes this effect because the data are effectively stacked to reinforce the signal from a specific target; however, it is not always possible to separate the signals. Another potential complication arises if the background conductivity is high because the coupling between the transmitter and the target will be different from the free-space scenario (completely resistive background) and may also change as a function of delay time. However, a completely resistive background is a valid model in many areas of the world at which deep exploration is ongoing and/or inevitable such as in the Canadian Shield and in Sudbury, Canada.

The overall multiple-transmitter concepts discussed in this paper are not reliant on the limitations of the dipole model discussed above. More sophisticated models (e.g., nondipole targets and conductive layered earth) could be used to derive coupling coefficients and to model the data to derive the optimal composite transmitter. Alternatively, the multitransmitter data could be input into a large $3 \mathrm{D}$ inversion. The potential advantage of using a general 3D inversion is high because it can be used to characterize the entire subsurface, not just for target detection/characterization. However, in their current state, large 3D inversions are not easily used due to their associated cost, turnaround time, and general lack of availability.

Logistically speaking, the optimum way to collect multitransmitter data would be with a hybrid ground/airborne system; the ground receivers can be laid out, and then an airborne transmitter is flown. If an airborne transmitter is not possible or feasible, then using small, multiturn, easily movable, ground loops would be recommended. To reduce surveying time and cost, it would be beneficial to lay many or all of the receivers at the same time, so as to reduce the amount of times the transmitter needs to be reflown in the airborne case or moved in the ground case. This could be achieved with a distributed array ground receiver system such as the one developed by Golden et al. (2006). There are no commercialy distributed array EM systems currently available, but this deficiency should be rectified soon. One further manner in which the logistics can be improved is by reducing the amount of data collected through the use of reciprocity. Lymburner and Smith (2015) show that in the field example presented here, the data density (and thus collection time) could be reduced by $28.75 \%$ by applying the principles of reciprocity. Alternatively, the reciprocal data could be included in the summation process (i.e., forming the composite transmitter) to increase the $\mathrm{S} / \mathrm{N}$ of the data as was done in this study.

One missing piece of information not discussed in this work is the conductivity of the target. This can be determined through decay rate analysis in the time domain (in-phase/quadrature ratio in the frequency domain) or through the use of conductivity-depth-imaging algorithms. The benefit of the method presented here is that once the high-S/N composite transmitter data are created, they can be used in standard EM data processing and interpretation schemes.

\section{CONCLUSION}

Using multiple transmitter locations has the advantage of ensuring strong coupling between the transmitter and the target(s). Furthermore, if the independent transmitter data are summed into a single composite transmitter, the $\mathrm{S} / \mathrm{N}$ can be drastically increased, allowing for deeper and more focused exploration. However, the optimal manner in which the data are summed depends on the location and orientation of the target(s). By discretizing the subsurface and possible target orientations, the approximate location and orientation can be found by comparing the measured response with the synthetic look-up table response of a target with a known location and orientation. Using synthetic examples, we show that this methodology is robust and can accurately determine the location and orientation of discrete target(s) embedded within a resistive medium. Once this is known, the multiple transmitter data can be summed into a single high-S/N composite transmitter. In a ground time-domain EM field test, 23 transmitter positions were used and a shallow target could be identified using the developed methodology. The produced composite transmitter data were considerably easier to interpret and had a higher signal amplitude than the data from any one single transmitter.

The work presented here provides an alternative strategy for the inevitable deeper exploration, which will be required as shallow deposits are exhausted. Rather than using very large magnetic moment ground loops, many smaller moment loops can be used and through summation become significantly larger than any ground loop. Future work aims to test and modify the methodology to work on targets embedded within a conductive half-space and/or layered earth.

\section{ACKNOWLEDGMENTS}

We are grateful to the following for financial support of this research: NSERC, Vale, Sudbury Integrated Nickel Operations, a Glencore Company, Wallbridge Mining, KGHM International, and the Centre for Excellence in Mining Innovation. M. Kolaj is grateful for an NSERC Alexander Graham Bell scholarship and an SEG George V. Keller Scholarship.

\section{APPENDIX A DIPOLE LOOK-UP TABLE}

The dipole look-up table matrix (L) solves for the three-component magnetic field using the dipole equation (Grant and West, 1965; Smith 2001):

$$
\mathbf{L}\left(\mathbf{r}_{d}, \mathbf{m}_{\theta}\right)=\frac{1}{4 \pi\left|\mathbf{r}_{d}-\mathbf{s}\right|^{3}}\left[\frac{3 \mathbf{m}_{\theta} \cdot\left(\mathbf{r}_{d}-\mathbf{s}\right)}{\left|\mathbf{r}_{d}-\mathbf{s}\right|^{2}}\left(\mathbf{r}_{d}-\mathbf{s}\right)-\mathbf{m}_{\theta}\right],
$$

where $\mathbf{r}_{d}$ is the position vector of the dipole, $\mathbf{m}_{\theta}$ is the unit vector parallel to the direction of the dipole (calculated from the strike and dip of the dipole), and $\mathbf{s}$ is the position vector of the station locations. In our implementation, $\mathrm{L}$ is precalculated for a set of $\mathbf{r}_{d}$ (possible target locations) and $\mathbf{m}_{\theta}$ (possible target orientations) and is normalized to \pm 1 . The end result is a $4 \mathrm{D}$ array of size $(i, j, 3$, and $m$ ), where $i$ is the total number of station locations where the magnetic field is to be calculated, $j$ is the total number of possible dipole orientations $\left(\mathbf{m}_{\theta}\right), 3$ corresponds to the three components of the 
magnetic field, and $m$ is the total number of possible dipole locations $\left(\mathbf{r}_{d}\right)$. In this work, a best-fitting dipole is calculated for each $\mathbf{r}_{d}$ using equation 2 .

\section{REFERENCES}

Golden, H., T. Herbert, and A. Duncan, 2006, GEOFERRET: A new distributed system for deep-probing TEM surveys: Presented at 76th Annual International Meeting in the Workshop on Uranium Exploration, SEG.

Grant, F. S., and G. F. West, 1965, Interpretation theory in applied geophysics: McGraw-Hill.

Lymburner, J., and R. Smith, 2015, A procedure for collecting electromagnetic data using multiple transmitters and receivers capable of deep and focussed exploration: Geophysics, 80, no. 1, E1-E10, doi: 10.1190/ geo2014-0251.1.

Nabighian, M. N., and J. C. Macnae, 1991, Time domain electromagnetic prospecting methods, in M. N. Nabighian, ed., Electromagnetic methods in applied geophysics: Applications, Parts A and B: SEG Investigations in Geophysics 3, 427-520.

Palacky, G. J., and G. F. West, 1991, Airborne electromagnetic methods, in M. N. Nabighian, ed., Electromagnetic methods in applied geophysics: Applications, Part A and B: SEG Investigations in Geophysics 3, 811879.

Pasion, L., and D. Oldenburg, 2001, A discrimination algorithm for UXO using time domain electromagnetics: Journal of Environmental and Engineering Geophysics, 6, 91-102, doi: 10.4133/JEEG6.2.91.

Polzer, B., J. Macnae, Y. Lamontagne, and R. Koch, 1989, Lateral resolution enhancement of TEM data by correlation processing: 59th Annual International Meeting, SEG, Expanded Abstracts, 182-184, doi: 10 $.1190 / 1.1889600$.

Powell, B., 1990, Large loop EM surveys in the Athabasca Basin, in L. S. Beck, and C. T. Harper, eds., Modern exploration techniques: Saskatchewan Geological Society, 74-93.
Powell, B., G. Wood, and L. Bzdel, 2007, Advances in geophysical exploration for uranium deposits in the Athabasca Basin, in B. Milkereit, ed., Proceedings of Exploration 07: Fifth Decennial International Conference on Mineral Exploration, Decennial Mineral Exploration Conferences, 771-790.

Sattel, D., and J. Reid, 2006, Modelling of airborne EM anomalies with magnetic and electric dipoles buried in a layered earth: Exploration Geophysics, 37, 254-260, doi: 10.1071/EG06254.

Smith, R. S., 2001, Tracking the transmitting-receiving offset in fixed-wing transient EM systems: Methodology and application: Exploration Geophysics, 32, 14-19, doi: 10.1071/EG01014.

Smith, R., D. Fountain, and M. Allard, 2003, The MEGATEM fixed-wing transient EM system applied to mineral exploration: A discovery case history: First Break, 21, 71-75.

Smith, R. S., and A. S. Salem, 2007, A discrete conductor transformation of airborne electromagnetic data: Near Surface Geophysics, 5, 87-95, doi 10.3997/1873-0604.2006021.

Smith, R., and A. Volkovitsky, 2014, Airborne electromagnetic prospecting systems, in A. A. Kaufman, and M. Oristaglio, eds., Principles of electromagnetic methods in surface and borehole geophysics: Methods in Geochemistry and Geophysics, vol. 45, 679-709.

Snyder, D. D., and C. P. Oden, 2012, Optimizing the MetalMapper for static "cued ID" measurements over UXO: Presented at the Symposium on the Application of Geophysics to Engineering and Environmental Problems, 333-347.

Walker, P., and G. F. West, 1991, A robust integral equation solution for electromagnetic scattering by a thin plate in conductive media: Geophysics, 56, 1140-1152, doi: 10.1190/1.1443133.

Zhdanov, M. S., 2010, Electromagnetic geophysics: Notes from the past and the road ahead: Geophysics, 75, no. 5, 75A49-75A66, doi: 10.1190/1 .3483901 . 